\title{
Single-Cycle Autocorrelation in Attosecond Coherent Nanotransport
}

\author{
M. Ludwig ${ }^{1}, T . \mathrm{Rybka}^{1}, F$. Ritzkowsky ${ }^{1}$, D.-C. Marinica ${ }^{2}, A$. Borissov $^{2}$, \\ $G$. Aguirregabiria ${ }^{3,4}, J$. Aizpurua ${ }^{3,4}, A$. Leitenstorfer ${ }^{1}$, and $D$. Brida ${ }^{1, *}$ \\ ${ }^{1}$ Department of Physics, University of Konstanz, D-78457 Konstanz, Germany \\ ${ }^{2}$ Institut des Sciences Moléculaires d'Orsay, CNRS-Université Paris-Sud, 91405 Orsay Cedex, France \\ ${ }^{3}$ Material Physics Center CSIC-UPV/EHU, 20018 Donostia-San Sebastián, Spain \\ ${ }^{4}$ Donostia International Physics Center DIPC, 20018 Donostia-San Sebastián, Spain
}

\begin{abstract}
We employ a pair of single-cycle near-infrared pulses to control coherent transfer of single electrons between the contacts of a plasmonic nanocircuit. As a result, attosecond and highly nonlinear phenomena occur at $\mathrm{pJ}$ pulse energies.
\end{abstract}

\section{Introduction}

Recently, we demonstrated that single-cycle pulses of minute energy content may result in extremely nonlinear optical phenomena at the nanoscale by exploiting an electronic circuit with a few-nanometer gap between the tips of an optical antenna. The strong electrical bias provided by the field contained in ultrashort optical pulses was harnessed to drive tunneling and ballistic acceleration of electrons to generate a current through the free-space gap with $\mathrm{PHz}$ bandwidth [1]. This non-perturbative process is fully coherent with the driving radiation and occurs within a half-cycle of the near-IR carrier wavelength. In this work, we further explore this concept by gaining direct temporal information via interferomeric autocorrelation measurements with two identical replicas of truly single-cycle driving pulses.

\section{Single-Cycle Autocorrelations with Carrier Envelope Phase Dependence}

Figure 1(a) depicts the scheme of the experimental technique. The pulses are set at a variable delay by a Mach-Zehnder interferometer and then focused tightly onto a nanocircuit equipped with a single bowtie nanoantenna. The circuit (Fig. 1(b)) consists of an open junction of approximately $6 \mathrm{~nm}$ between two gold tips of a bowtie antenna fabricated via electron beam lithography. Under a quasi-static bias, this structure exhibits a tunneling-like current-voltage (I-V) characteristics that is antisymmetric and 
highly nonlinear. The bowtie design allows sub-wavelength concentration of the electric field of the laser pulse while preserving its single-cycle duration.

a)

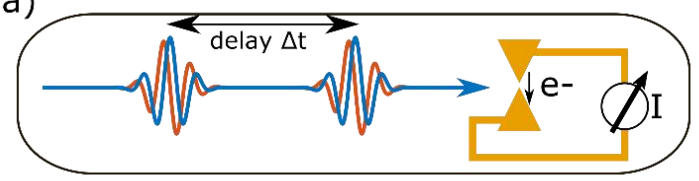

b)

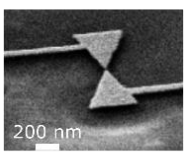

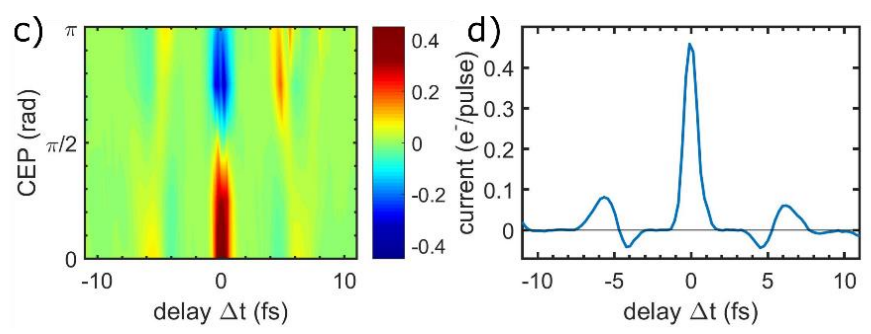

Fig. 1. (a) Conceptual sketch of the experiment with two single-cycle light pulses at delay time $\Delta t$ focused on the nanogap of the circuit. The carrier-envelope phase of the biasing pulses can selected arbitrarily. (b) Scanning electron micrograph of the nanocircuit employed in the experiments, consisting of an Au optical antenna with electrical leads on a fused-silica substrate. (c) Colormap of nanotunneling current as a function of delay between the two driving pulses and as a function of their carrier-envelope phase. (d) Autocorrelation trace as a function of time delay $\Delta t$ measured for a carrier-envelope phase of 0 (i.e. cosine-like pulses), corresponding to maximum total current.

Our $80 \mathrm{MHz}$ Er:fiber laser system [2] generates 4.2-fs single-cycle pulses at a carrier wavelength of $1325 \mathrm{~nm}$ spanning from $800 \mathrm{~nm}$ to $2200 \mathrm{~nm}$ [2]. A passive phase-locking scheme is implemented to allow arbitrary control of the carrier-envelope phase without affecting the temporal duration of the pulse [1]. These electrical transients provide a strong and ultrafast bias for driving of electronic transport across the junction. Due to the strong nonlinearity of the I-V characteristics, an optically-induced symmetry break occurs in the electric transport which leads to a net current that solely depends on the carrier-envelope phase [1]. For the autocorrelation measurements, the current generated at the nanojunction of the circuit is recorded as a function of both the delay between the two pulse replicas and their carrier-envelope phase. The full dataset for the interferometric autocorrelation as a function of the carrier-envelope phase is shown in Fig. 1(c) as a color map. At perfect temporal overlap (delay $=0 \mathrm{fs}$ ) the optical power amounts to $4 \mathrm{~mW}$, still corresponding to a minute driving pulse energy of $50 \mathrm{pJ}$. Fig. $1(d)$ shows the current recorded as a function of the delay for a carrier-envelope-phase set to 0 . This measurement is indeed an interferometric autocorrelation based on a nonperturbative nonlinear process for the single-cycle pulse. Background-free currents of up to $5 \mathrm{pA}$ can be detected in the case of $\Delta \mathrm{t}=0 \mathrm{fs}$. This value corresponds to approximately one electron being transported every second pulse. The temporal width of the central maximum in the current measured for cosine-shaped pulses $(\mathrm{CEP}=0)$ is found to be less than 1 femtosecond which is well below the duration of a half-cycle of the driving field. This finding places the average transfer time of an electron between the two contacts in the attosecond range. Our results are a clear and direct indicator for the fact that the electronic transport at the nanojunction is fully coherent with the 
driving pulses and takes place on timescales shorter than a half-cycle of the driving field.

\section{Model of the Ultrafast Nanocurrents}

Fig. 2 shows the results of the Time Dependent Density Functional Theory calculations of the strong field ionization in plasmonic gaps. The theoretical analysis has been performed for a model system composed by two identical gold cylinders of $5 \mathrm{~nm}$ radius separated by $6 \mathrm{~nm}$ gap. Fig 2(b) shows the time evolution of the electric field in the center of the gap (normalized to 1). In the (c) and (d) panels we plot the electronic current along the dimer $\mathrm{x}$-axis as a function of time, for two different pulse intensities.

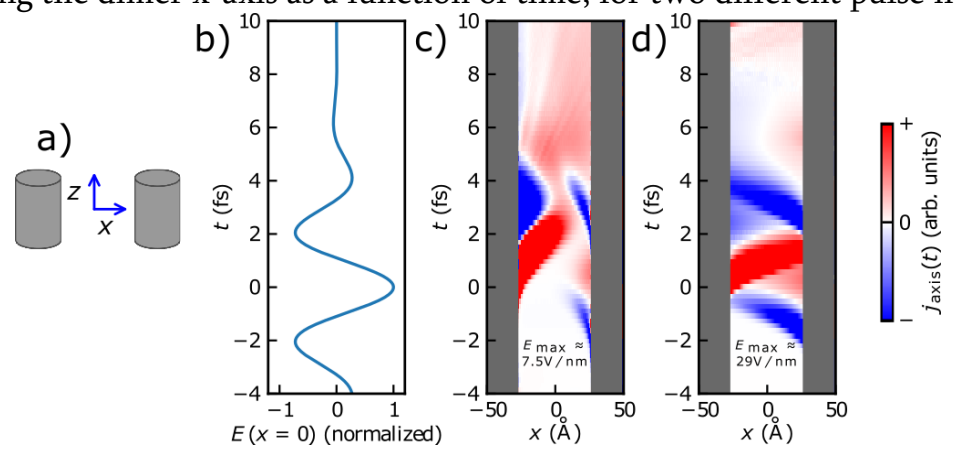

Fig. 1. (a) Sketch of the model structure employed for the calculations. (b)Electric field profile of the single cycle optical transient. (c,d) Colormap depicting the carrier density within the gap as a function of the time delay (vertical axis) for an optical field of $7.5 \mathrm{~V} / \mathrm{nm}$ and $29 \mathrm{~V} / \mathrm{nm}$, respectively.

The emitted electrons cross the junction resulting in the charge transfer between the cylinders. Interestingly, the oscillating electric field in the gap results in the quiver motion of the emitted electrons that is particularly visible for low intensity of the incident pulse (central panel) owing to the small quiver amplitude. At higher fields the transport within the gap occurs within one optical cycle of the driving field.

\section{Conclusions}

In summary, we have performed interferometric autocorrelation measurements with single-cycle pulses that exploit the electric currents coherently driven at the gap of a single nanodevice by optical pulse with $\mathrm{pJ}$ energies. The full width at half maximum of the current autocorrelation amounts to less than one femtosecond, demonostrating that we can transfer individual electrons between the two contacts on an attosecond time scale. In the future, we are aiming at a regime where the Coulomb interaction between electrons becomes important at truly atomic time and length scales.

\section{References}

1. T. Rybka, M. Ludwig, M. F. Schmalz, V. Knittel, D. Brida und A. Leitenstorfer, Nature Photon. 10, 667-670 (2016).

2. D. Brida, G. Krauss, A. Sell and A. Leitenstorfer, Laser Phot. Rev. 8, 409-428 (2014). 